\title{
General formulation of digital in-line holography from correlation with a chirplet function
}

\author{
S. Coëtmellec \\ coetmellec@coria.fr
}

\section{N. Verrier}

\section{Brunel \\ D. Lebrun}

\begin{abstract}
Département d'Optique, UMR-6614 CORIA, Av. de l'Université, 76801 Saint-Etienne du Rouvray cedex, France

Département d'Optique, UMR-6614 CORIA, Av. de l'Université, 76801 Saint-Etienne du Rouvray cedex, France

Département d'Optique, UMR-6614 CORIA, Av. de l'Université, 76801 Saint-Etienne du Rouvray cedex, France

Département d'Optique, UMR-6614 CORIA, Av. de l'Université, 76801 Saint-Etienne du Rouvray cedex, France
\end{abstract}

Digital in-line holography is revisited to propose a mathematical model that describes the recording-reconstruction process as a linear shift-invariant system with a pseudo-point spread function even when the images are out of the optimal plane in the sense of signal processing. A particular case is treated to show that the optimal plane is the best focus plane in the sense of optics. Next, an exact solution of the holographic reconstruction by correlation is given. By means of the previous results, we study the behavior of the result of the correlation function between the diffraction pattern function produced by an opaque disk and a chirplet function and between the diffraction pattern produced by a phase disk and the same chirplet function. [D0I: 10.2971/jeos.2010.10027]

Keywords: digital in-holography, correlation

\section{INTRODUCTION}

In nature, the class of chirp functions is one of the most important classes. A wide ranged domain of applications such as seismic signals, sonar, speech signals and images, ECG and radar signals are concerned with such functions [1]. The physical domain considered in this publication is physical optics and more precisely diffraction and interference phenomena where the linear frequency modulation (FM) chirp is preponderant $[2,3]$. To describe such physical processes, the mathematical tool used is an integral operator with a scaling chirp kernel. The scaling chirp kernel takes the following form [4]:

$$
h_{\gamma}(b, c)=\kappa(a) \exp \left[i \frac{(\xi-b)^{2}+(\eta-c)^{2}}{a^{2}}\right],
$$

where the multi-index parameter $\gamma=(a, b, c)$ is an element of the set $\Gamma=\mathbb{R}^{\star+} \times \mathbb{R}^{2} . \kappa(a)$ is an amplitude modifier. The variables $a$ and $(b, c)$ are the scale and shift parameters. From a signal processing point of view, the integral operator appears as an inner product such as:

$$
\langle f, g\rangle=\iint f(\xi, \eta) \overline{g(\xi, \eta)} d \xi d \eta
$$

The upper bar denotes the conjugate complex. The observation of the diffraction kernels allow the authors of [4] to review diffraction and in-line holography within chirplet transformation. The technique is widely used because it is non-intrusive and it is an in situ method. With the advancement of computers and CCD devices, digital in-line holography has developed rapidly in the many areas such as surface contouring [3], shape deformation analysis [5], etc. The basic idea in digital in-line holography (DIH) is firstly to record the intensity distribution, with a CCD sensor, of the diffraction pattern of an object illuminated by a continuous or pulsed wave $[6,7,8]$. The second step is to reconstruct the image of this object by means of an operator. In this publication, two object types will be considered: an opaque disk and a phase disk for the transparent object. The choice of these objects is linked to the rotational invariance. It is then possible to compare theoretical developments and numerical, experimental results. It is clear that work presented here often applies to fluid flow seeded with small spherical particles. Generally, in the far field approximation, spherical particles and opaque disks give the same diffraction patterns [9]. Figure 1 represents the numerical and experimental set-up where all parameters are identified.

The field $\phi(x, y)$ in the plane of the CCD sensor located at the distance $z_{e}$ from the object is given by the Huygens-Fresnel

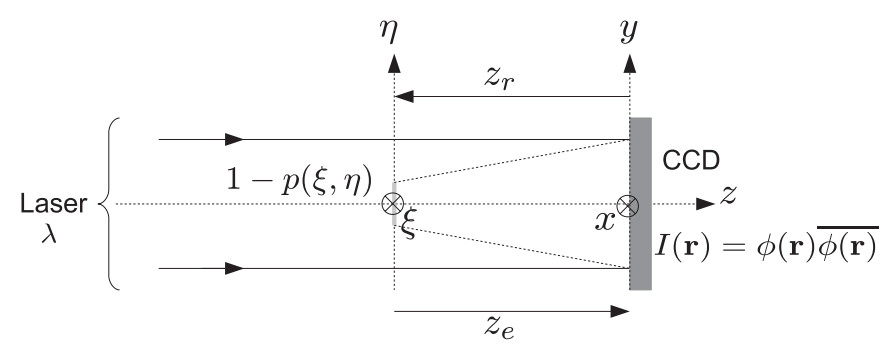

FIG. 1 Experimental set-up for digital in-line hologram recording and reconstruction by correlation. $z_{e}$ distance of recording, $z_{r}$ distance of the reconstruction. 
approximation as [7]:

$$
\begin{aligned}
\phi(\mathbf{r})= & \frac{\exp \left(i \frac{2 \pi}{\lambda} z_{e}\right)}{i \lambda z_{e}} \iint_{\mathbb{R}}[1-p(\xi, \eta)] \\
& \times \exp \left(i \frac{\pi}{\lambda z_{e}}\left[(\xi-x)^{2}+(\eta-y)^{2}\right]\right) d \xi d \eta,
\end{aligned}
$$

where $\mathbf{r}=(x, y)$ and $r=\sqrt{x^{2}+y^{2}}$. As in-line holography is considered, the phase term $\exp \left(i 2 \pi z_{e} / \lambda\right)$ can be omitted. The distribution $[1-p(\xi, \eta)]$ corresponds to the transmission function of the object and $\lambda$ is the wavelength. For an opaque disk, the distribution $p(\xi, \eta)$ in Eq. (3) is defined by:

$$
\Pi(\xi, \eta)=\mathbf{1}_{\mathcal{S}}
$$

Its value is equal to unity over the surface $\mathcal{S}$ defined by $\mathcal{S}=$ $\left\{(\xi, \eta) \mid \xi^{2}+\eta^{2} \leq d^{2} / 4\right\}$ and $d$ is the diameter of the disk. In the case of a phase disk, the function $p(\xi, \eta)$ is:

$$
[1-\exp (i \varphi)] \Pi(\xi, \eta)
$$

The intensity distribution $I(\mathbf{r})$ of the field $\phi(\mathbf{r})$ recorded by the CCD sensor is then:

$$
I(\mathbf{r})=\phi(\mathbf{r}) \overline{\phi(\mathbf{r})} .
$$

For an opaque disk, the intensity distribution is defined by:

$$
\begin{aligned}
I_{o}(\mathbf{r})=I=1-\frac{\pi d^{2}}{\lambda z} & \sin \left(\frac{\pi r^{2}}{\lambda z}\right) \frac{J_{1}\left(\frac{\pi d}{\lambda z} r\right)}{\left(\frac{\pi d}{\lambda z} r\right)} \\
& +\frac{1}{(\lambda z)^{2}}\left(\frac{\pi d^{2}}{2}\right)^{2} \frac{J_{1}^{2}\left(\frac{\pi d}{\lambda z} r\right)}{\left(\frac{\pi d}{\lambda z} r\right)^{2}} .
\end{aligned}
$$

But in the case of a phase disk, from [10] and [11], $I_{p}$ takes the following definition:

$$
\begin{gathered}
I_{p}(\mathbf{r})=I=1+\frac{\pi d^{2}}{\lambda z}\left[\sin \left(\frac{\pi r^{2}}{\lambda z}+\varphi\right)-\sin \left(\frac{\pi r^{2}}{\lambda z}\right)\right] \frac{J_{1}\left(\frac{\pi d}{\lambda z} r\right)}{\left(\frac{\pi d}{\lambda z} r\right)} \\
+\frac{2-2 \cos \varphi}{(\lambda z)^{2}}\left(\frac{\pi d^{2}}{2}\right)^{2} \frac{J_{1}^{2}\left(\frac{\pi d}{\lambda z} r\right)}{\left(\frac{\pi d}{\lambda z} r\right)^{2}}
\end{gathered}
$$

In the far field approximation, i.e. $\pi d^{2} /(2 \lambda z)<1$, the third term can be neglected. Figure 2 illustrates us a numerical example of diffraction patterns of an opaque disk (Figure 2(a)) and a phase disk (Figure 2(b)) recorded by a CCD sensor.

Let us recall that the intensity distribution may be represented as a bidimensional convolution between $\Pi(\mathbf{r})$, and the chirplet from Eq. (1), such as for the opaque disk, one has:

$$
\mathcal{I}_{o}(\mathbf{r})=1+\frac{i}{\lambda z_{e}} \Pi(\mathbf{r}) *\left[h_{z_{e}}(\mathbf{r})-\bar{h}_{z_{e}}(\mathbf{r})\right]
$$

with

$$
h_{z}(\mathbf{r})=\exp \left[i \frac{\pi r^{2}}{\lambda z}\right] .
$$

About the phase disk, the intensity distribution can be defined by:

$$
\mathcal{I}_{p}(\mathbf{r})=\mathcal{I}_{o}(\mathbf{r})-\frac{i}{\lambda z_{e}} \Pi(\mathbf{r}) *\left[h_{\varphi}(\mathbf{r})-\bar{h}_{\varphi}(\mathbf{r})\right]
$$

with

$$
h_{\varphi}(\mathbf{r})=\exp \left[i \frac{\pi r^{2}}{\lambda z}+i \varphi\right] .
$$

The symbol $*$ denotes the spatial 2D-convolution.
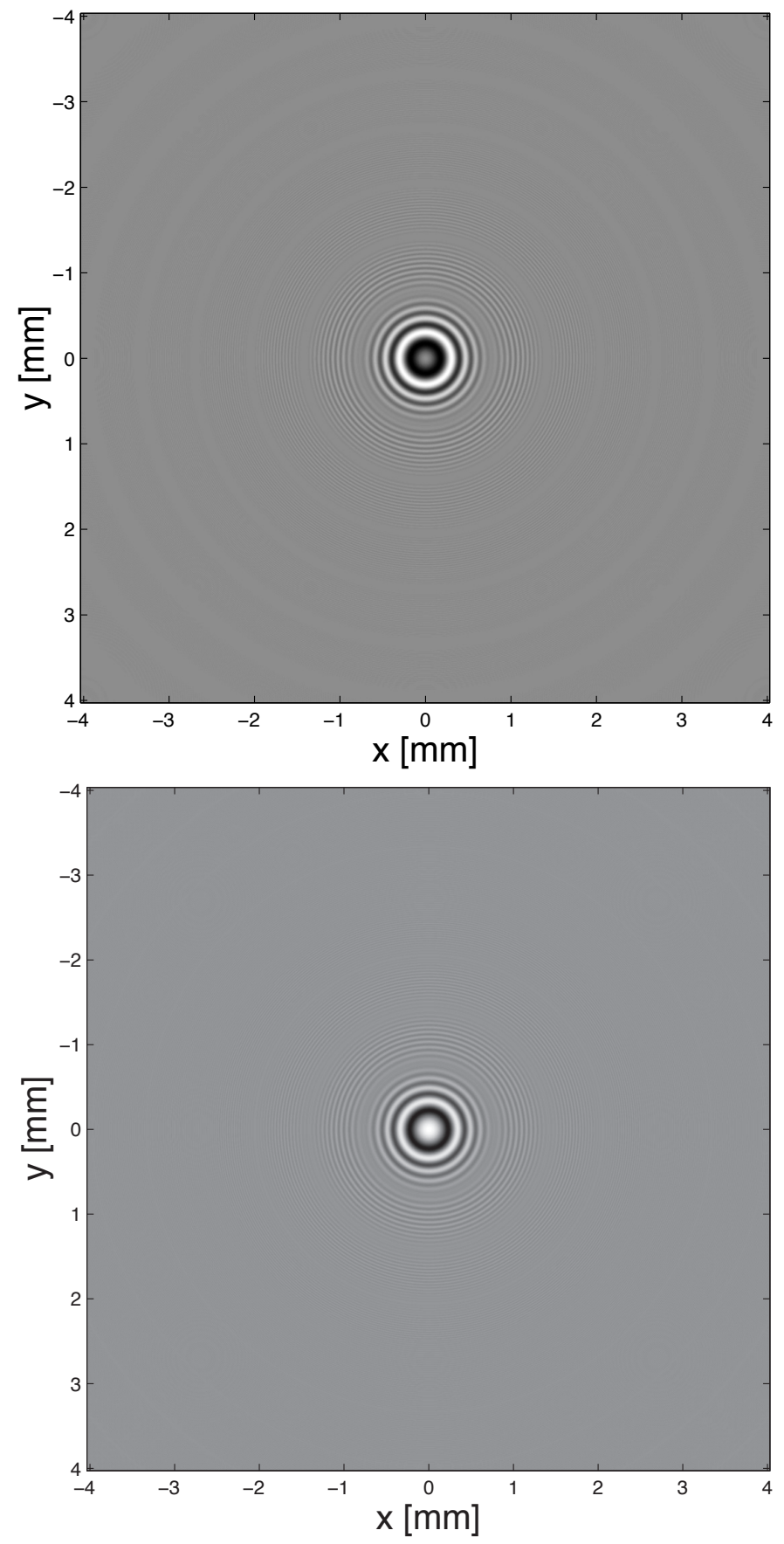

FIG. 2 Intensity distribution of the diffraction pattern of (a) an opaque disk, (b) a phase objet with $\varphi=0.354 \pi . d=100 \mu \mathrm{m}, \lambda=635 \mathrm{~nm}, z_{e}=100 \mathrm{~mm}, 1024 \times 1024$, pixel size $7.87 \mu \mathrm{m} \times 7.87 \mu \mathrm{m}$.

\subsection{Reconstruction process}

In [4], the authors demonstrate that the reconstruction of the image of the object is possible in the framework of the scaling chirp transformation. Nevertheless, the optical signal is sampled by a matrix sensor. Moiré effects may appear on the digitized diffraction pattern that can disturb the numerical reconstruction, in particular when a field of particles is considered [10]. Indeed, when we reconstruct the image of the particle, at the same time, the images of the moiré patterns are also reconstructed. The image of particles linked to moiré patterns appears in white gray level.

To minimize the moiré effect and to perform the numerical 
reconstruction, the authors of [12] used a real and spatial localized function. In the literature, these type of functions are also called Gaussian chirplet [1]. The digital reconstruction of the image of the object can be realized in many ways. Generally, the Fresnel integral is used first and other operators are also considered such as fractional Fourier transformation [13] or real Gaussian chirplet [14]. These transformations are convolution operators. Nevertheless, correlation techniques can be used in the DIH to reconstruct the image of the particle. Let us note $C_{a}$ the correlation between the intensity distribution $I(x, y)$ and a real Gaussian chirplet, denoted $\psi_{a}(x, y)$ and such that:

$$
C_{a}\left(I, \psi_{a}\right)=I(\mathbf{r}) \star \psi_{a}(\mathbf{r}) .
$$

Note that the correlation and convolution are similar in our case because all the functions are real and even. The reconstruction technique used here is based on a real Gaussian chirplet, kernel denoted $\psi_{a}(\mathbf{r})$, of zero mean value and given by:

$$
\psi_{a}(\mathbf{r})=\frac{1}{a^{2}}\left[\sin \left(\frac{r^{2}}{a^{2}}\right)-M_{\psi}\right] \exp \left[-\frac{r^{2}}{(a \sigma)^{2}}\right] .
$$

The $\sigma$ parameter is fixed to limit the moiré effects and assures the spatial localization of the chirplet. Generally, it is taken greater than 5 . The scale parameter, denoted $a$, allows the similarity between $\psi_{a}(\mathbf{r})$ and $I(\mathbf{r})$ to be increased. Its expression is defined as [15]:

$$
a^{2}=\frac{\lambda z_{r}}{\pi}
$$

and the zero mean value of $\psi_{a}$ is assured by:

$$
M_{\psi}=\frac{\sigma^{2}}{1+\sigma^{4}} .
$$

To simplify the further calculus, Eq. (14) can be expressed versus Eq. (12) as:

$$
\psi_{a}(\mathbf{r})=\left[-\frac{i}{2 a^{2}}\left(h_{z_{r}}-\bar{h}_{z_{r}}\right)-\frac{M_{\psi}}{a^{2}}\right] \exp \left[-\frac{r^{2}}{(a \sigma)^{2}}\right] .
$$

The parameter $z_{r}$ is the reconstruction distance as one can see in Figure 1. Consequently, the equality $z_{r}=z_{e}$ should be the best choice to apply metrologies on the intensity distribution obtained [14]. The reconstruction process is usually timeconsuming. However, various analysis techniques have been developed [16, 17]. But here, the aim is to propose a mathematical model that describes the recording-reconstruction inline holography process and to understand the influence of the parameters of the function $\psi_{a}(x, y)$. To numerically compute correlation Eq. (13), the fast Fourier transform (FFT) is usually used and the calculation process is:

$$
C_{a}\left(I, \psi_{a}\right)=\mathcal{F}^{-1}\left[\mathcal{F} I \times \mathcal{F} \psi_{a}\right],
$$

where $\mathcal{F}$ and $\mathcal{F}^{-1}$ are Fourier and inverse Fourier transformation operators. Figure 3 shows the reconstruction of an image of the objects from Figure 2.

The reconstruction of the image of the phase disk is different to the reconstruction of the image of the opaque disk. Indeed, the phase disk is a transparent phase plate. The reconstruction process allows us to obtain a different linear chirp from the opaque disk and the value at the origin is the smallest. These images have been obtained by using Eq. (18). Let

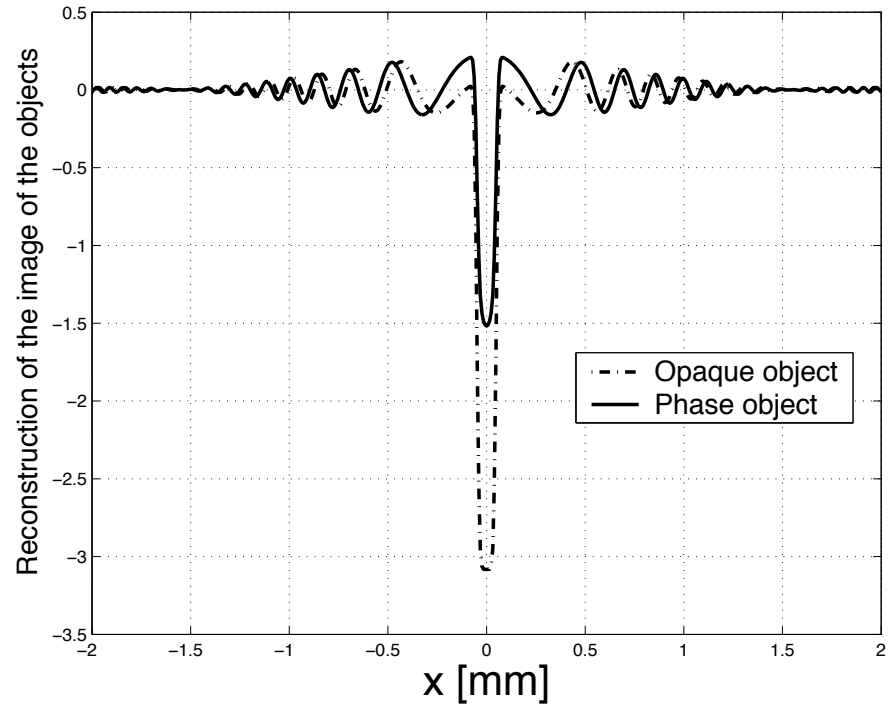

FIG. 3 Profile of the reconstructions of the image of the objects for $z_{r}=z_{e}=100 \mathrm{~mm}$ from the intensity distribution of the diffraction pattern of a $100 \mu \mathrm{m}$ disk localized at $100 \mathrm{~mm}$ of the CCD sensor, $\lambda=632.8 \mathrm{~nm}$ and $\sigma=6$.

us recall the important fact that the inner product of real Gaussian chirplets is not equal to the Kronecker delta function. It is precisely the difference between Fresnel or fractional Fourier transformation and real Gaussian chirplets transformation. The first transformations have orthogonal kernels. Consequently, it is important here to understand the influence of the parameters $a$ and $\sigma$. For this reason, an analytical development is necessary.

\subsection{Preliminary developments}

\subsubsection{Case of opaque disk}

As the convolution is associative and distributive, the correlation between the intensity distribution $\mathcal{I}_{0}$ and the wavelet $\psi_{a}$ can be written:

$$
\begin{gathered}
C_{a}\left(\mathcal{I}_{0}, \psi_{a}\right)=\frac{1}{2 \lambda z_{e} a^{2}} \Pi(\mathbf{r}) *\left[T_{1}-T_{2}-T_{3}+T_{4}\right] \\
-\frac{i M_{\psi}}{\lambda z_{e} a^{2}} \times \Pi(\mathbf{r}) * T_{5},
\end{gathered}
$$

with the five functions $T_{1, \ldots, 5}$, defined by Eqs. (20):

$$
\begin{aligned}
& T_{1}=h_{z_{e}} *\left[g \cdot h_{z_{r}}\right], \\
& T_{2}=h_{z_{e}} *\left[g \cdot \bar{h}_{z_{r}}\right], \\
& T_{3}=\bar{h}_{z_{e}} *\left[g \cdot h_{z_{r}}\right], \\
& T_{4}=\bar{h}_{z_{e}} *\left[g \cdot \bar{h}_{z_{r}}\right],
\end{aligned}
$$

and

$$
T_{5}=\left[h_{z_{e}}-\bar{h}_{z_{e}}\right] * g
$$

The function denoted $g$ corresponds to the Gaussian function of the Gaussian chirplet in Eq. (14) and defined by $\exp \left(-r^{2} /(a \sigma)^{2}\right)$. The mathematical development of the expressions of the functions $T_{1, \ldots, 5}$ is given in Appendix A. 


\begin{tabular}{|c|c|c|c|c|c|}
\hline$l$ & $K_{l}$ & $\alpha_{l}$ & $\beta_{l}$ & $M_{l}$ & $\varphi_{l}$ \\
\hline 1 & $\frac{-1}{a^{2} M_{1}}$ & $\frac{1}{\sigma^{2} M_{1}^{2} a^{2}}$ & $\frac{1}{M_{1}^{2} a^{2}}\left(1+\frac{z_{e}}{z_{r}}\left(1+\sigma^{-4}\right)\right)$ & $\left|\left(1+\frac{z_{e}}{z_{r}}\right)+i \frac{1}{\sigma^{2}} \frac{z_{e}}{z_{r}}\right|$ & $\arctan \left(\frac{1}{\sigma^{2}} \frac{z_{e} / z_{r}}{1+z_{e} / z_{r}}\right)$ \\
\hline 2 & $\frac{-1}{a^{2} M_{2}}$ & $\frac{1}{\sigma^{2} M_{2}^{2} a^{2}}$ & $\frac{1}{M_{2}^{2} a^{2}}\left(1-\frac{z_{e}}{z_{r}}\left(1+\sigma^{-4}\right)\right)$ & $\left|\left(1-\frac{z_{e}}{z_{r}}\right)+i \frac{1}{\sigma^{2}} \frac{z_{e}}{z_{r}}\right|$ & $= \begin{cases}\arctan \left(\frac{1}{\sigma^{2}} \frac{z_{e} / z_{r}}{1-z_{e} / z_{r}}\right), & 1-z_{e} / z_{r}>0, \\
\arctan \left(\frac{1}{\sigma^{2}} \frac{z_{e} / z_{r}}{1-z_{e} / z_{r}}\right)+\pi, & 1-z_{e} / z_{r}<0 .\end{cases}$ \\
\hline 3 & $\frac{2 M_{\psi}}{a^{2} M_{3}}$ & $\frac{1}{\sigma^{2} M_{3}^{2} a^{2}}$ & $\frac{1}{\sigma^{4} M_{3}^{2} a^{2}} \frac{z_{e}}{z_{r}}$ & $1+i \frac{1}{\sigma^{2}} z_{e}$ & $\arctan \left(\frac{1}{\sigma^{2}} \frac{z_{e}}{z_{r}}\right)$ \\
\hline
\end{tabular}

TABLE 1 Coefficients of the pseudo-point spread function $\mathcal{K}_{o}$.

\subsubsection{Case of phase disk}

The correlation between the intensity distribution $\mathcal{I}_{p}$ and the wavelet $\psi_{a}$ is the addition of the term defined by Eq. (19):

$$
\begin{gathered}
C_{a}\left(\mathcal{I}_{p}, \psi_{a}\right)=C_{a}\left(\mathcal{I}_{o}, \psi_{a}\right)+\frac{1}{2 \lambda z_{e} a^{2}} \Pi(\mathbf{r}) *\left[\Omega_{2} \Omega_{3}-\Omega_{1}-\Omega_{4}\right] \\
+\frac{i M_{\psi}}{\lambda z_{e} a^{2}} \Pi(\mathbf{r}) * \Omega_{5}
\end{gathered}
$$

with the five functions $\Omega_{1, \ldots, 5}$, defined by Eqs. (22):

$$
\begin{aligned}
& \Omega_{1}=T_{1} \cdot \exp [i \varphi], \\
& \Omega_{2}=T_{2} \cdot \exp [i \varphi], \\
& \Omega_{3}=T_{3} \cdot \exp [-i \varphi], \\
& \Omega_{4}=T_{4} \cdot \exp [-i \varphi],
\end{aligned}
$$

and

$$
\Omega_{5}=\left[h_{\varphi}-\bar{h}_{\varphi}\right] * \exp \left(-\frac{r^{2}}{(a \sigma)^{2}}\right) .
$$

\section{OUT OF OPTIMAL PLANE PSEUDO-POINT SPREAD FUNCTION}

\subsection{Main result}

In order to predict the reconstruction process on holographic images by DIH, it is important to describe the intensity distribution of out of optimal plane images (i.e. $z_{r} \neq z_{\ell}$, see Figure 1). First, the aim of this section consists of finding the analytical evolution of the pseudo-point spread function, denoted $\mathcal{K}_{o}$ for an opaque disk and $\mathcal{K}_{p}$ for a phase disk, when the reconstruction reaches the best plane at distance $z_{r}$.

\subsubsection{Case of opaque disk}

To determine the pseudo-point spread function, the functions $T_{1}$ and $T_{4}$ must be added on the one hand and $T_{2}$ added to $T_{3}$ on the other hand to obtain sinusoidal functions. Let us define $F_{1}=T_{1}+T_{4}, F_{2}=T_{2}+T_{3}$ and $F_{3}=T_{5}$. The definition of the correlation function becomes then:

$$
C_{a}\left(\mathcal{I}_{o}, \psi_{a}\right)=\Pi *\left(F_{1}+F_{2}+F_{3}\right)=\Pi * \mathcal{K}_{o} .
$$

The pseudo-point spread function $\mathcal{K}_{o}=F_{1}+F_{2}+F_{3}$ is given by:

$$
\begin{aligned}
\mathcal{K}_{o}= & K_{1} \exp \left[-\alpha_{1} r^{2}\right] \sin \left[\beta_{1} r^{2}-\varphi_{1}\right] \\
& +K_{2} \exp \left[-\alpha_{2} r^{2}\right] \sin \left[\beta_{2} r^{2}+\varphi_{2}\right] \\
& +K_{3} \exp \left[-\alpha_{3} r^{2}\right] \cos \left[\beta_{3} r^{2}-\varphi_{3}\right] .
\end{aligned}
$$

The coefficients $K_{l}, \alpha_{l}, \beta_{l}$ and $\varphi_{l}$ are given in Table 1 . It should be noted that the coefficients $K_{l}$ are the product of the coefficients $1 /\left(\lambda z_{e}\right)$ of Eq. (19) by the coefficients $\lambda z_{e}$ of the functions $T_{1, \ldots, 5}$. The other coefficients allow us to obtain the sinusoidal functions of the pseudo-point spread function $\mathcal{K}_{o}$.

\subsubsection{Case of phase disk}

The reconstruction of a phase disk is based on the reconstruction of an opaque disk with an additional term such as:

$$
C_{a}\left(\mathcal{I}_{p}, \psi_{a}\right)=C_{a}\left(\mathcal{I}_{o}, \psi_{a}\right)+\Pi * \mathcal{K}_{p}
$$

with

$$
\begin{aligned}
\mathcal{K}_{p}= & -K_{1} \exp \left[-\alpha_{1} r^{2}\right] \sin \left[\beta_{1} r^{2}-\left(\varphi_{1}-\varphi\right)\right] \\
& -K_{2} \exp \left[-\alpha_{2} r^{2}\right] \sin \left[\beta_{2} r^{2}+\left(\varphi_{2}-\varphi\right)\right] \\
& +K_{3} \exp \left[-\alpha_{3} r^{2}\right] \sin \left[\beta_{3} r^{2}-\left(\varphi_{3}-\varphi\right)\right] .
\end{aligned}
$$

\subsection{Particular case of the reconstruction of an image of the object}

In the reconstruction process, an image of the object is numerically reconstructed when $z_{r}$ tends to $z_{e}$. To compare the results in this paper with [14] and by taking into account the relation $\arctan \left(1 /\left(2 \sigma^{2}\right)\right)=\pi / 2-\arctan \left(2 \sigma^{2}\right)$, we have:

$$
\begin{aligned}
\lim _{\frac{z_{e}}{z_{r}} \rightarrow 1} \mathcal{K}_{o}=\frac{\sigma^{2}}{a^{2}} & \frac{1}{\sqrt{1+4 \sigma^{4}}} \exp \left[-\frac{\sigma^{2}}{1+4 \sigma^{4}} \frac{r^{2}}{a^{2}}\right] \\
& \times \cos \left[\frac{1+2 \sigma^{4}}{1+4 \sigma^{4}} \frac{r^{2}}{a^{2}}+\arctan \left(2 \sigma^{2}\right)\right] \\
& -\frac{\sigma^{2}}{a^{2}} \exp \left[-\sigma^{2} \frac{r^{2}}{a^{2}}\right] \cos \left[\frac{r^{2}}{a^{2}}\right] \\
+ & \frac{\sigma^{2}}{a^{2}} \frac{2 M_{\psi}}{\sqrt{1+\sigma^{4}}} \exp \left[-\frac{\sigma^{2}}{1+\sigma^{4}} \frac{r^{2}}{a^{2}}\right] \\
& \times \cos \left[\frac{1}{1+\sigma^{4}} \frac{r^{2}}{a^{2}}-\arctan \left(\frac{1}{\sigma^{2}}\right)\right] .
\end{aligned}
$$

Eq. (27) demonstrates that none of the functions obtained correspond to Dirac's impulse distribution $\delta(r)$. Consequently, the image of the object defined by the transmittance function $\Pi(\mathbf{r})$ is not obtained. This demonstration shows that this recording-reconstruction process by means of the real Gaussian chirplet defined by Eq. (14) is not a numerical refocusing of the object. It is the same case for the phase disk where we obtain:

$$
\lim _{\frac{z_{e}}{z_{r}} \rightarrow 1} \mathcal{K}_{p}=\lim _{\frac{z_{e}}{z_{r}} \rightarrow 1} \mathcal{K}_{o}-\mathcal{L}_{p},
$$


with

$$
\begin{aligned}
\mathcal{L}_{p}= & \frac{\sigma^{2} / a^{2}}{\sqrt{1+4 \sigma^{4}}} \exp \left[-\frac{\sigma^{2} / a^{2}}{1+4 \sigma^{4}} r^{2}\right] \\
& \times \cos \left[\frac{1+2 \sigma^{4}}{1+4 \sigma^{4}} \frac{r^{2}}{a^{2}}+\arctan \left(2 \sigma^{2}\right)+\varphi\right] \\
& -\frac{\sigma^{2}}{a^{2}} \exp \left[-\sigma^{2} \frac{r^{2}}{a^{2}}\right] \cos \left[\frac{r^{2}}{a^{2}}-\varphi\right] \\
+ & \frac{\sigma^{2}}{a^{2}} \frac{2 M_{\psi}}{\sqrt{1+\sigma^{4}}} \exp \left[-\frac{\sigma^{2}}{1+\sigma^{4}} \frac{r^{2}}{a^{2}}\right] \\
& \times \cos \left[\frac{1}{1+\sigma^{4}} \frac{r^{2}}{a^{2}}-\left(\arctan \left(\sigma^{2}\right)-\varphi\right)\right] .
\end{aligned}
$$

Consequently, a detailed study on the correlation product must be realized.

\section{THEORETICAL DEVELOPMENT OF THE CORRELATION PRODUCT OF I BY $\psi_{a}$}

\subsection{Main results in general case $\left(z_{r} \neq z_{e}\right.$ and $\sigma$ finite) for the opaque disk}

Let us consider the integral form of the convolution product of the Eq. (23) defined by :

$$
C_{a}\left(\mathcal{I}_{o}, \psi_{a}\right)=\Pi * \mathcal{K}_{o}=\iint_{\mathbb{R}^{2}} \Pi(\xi, \eta) \cdot \mathcal{K}_{o}(x-\xi, y-\eta) d \xi d \eta .
$$

As $\mathcal{K}_{o}=F_{1}+F_{2}+F_{3}$

$$
C_{a}\left(\mathcal{I}_{0}, \psi_{a}\right)=\Pi *\left(F_{1}+F_{2}+F_{3}\right)=\mathcal{C}_{1}+\mathcal{C}_{2}+\mathcal{C}_{3},
$$

with $\mathcal{C}_{1}=\Pi * F_{1}, \mathcal{C}_{2}=\Pi * F_{2}$ and $\mathcal{C}_{3}=\Pi * F_{3}$ ( $\Pi$ corresponds to Eq. (4)). The functions $F_{l}$ have similar mathematical structure. The estimation of $\mathcal{C}_{2}$ and $\mathcal{C}_{3}$ will thus be analogous to the estimation of $\mathcal{C}_{1}$. The mathematical development of $\mathcal{C}_{1}$ is given in Appendix B and is equal to:

$$
\begin{aligned}
\mathcal{C}_{1}=K_{1} & \frac{\pi d^{2}}{2} \exp \left[-\alpha_{1}\left(r^{2}+d^{2} / 8\right)\right] \cdot\left|W_{00}\left(r, f_{1}\right)\right| \cdot \\
& \sin \left[\beta_{1}\left(r^{2}+d^{2} / 8\right)-\varphi_{1}+\arg \left(W_{00}\left(r, f_{1}\right)\right)\right] .
\end{aligned}
$$

The functions $\mathcal{C}_{2}$ and $\mathcal{C}_{3}$ are deduced from the structure of $\mathcal{C}_{1}$, then:

$$
\begin{aligned}
& \mathcal{C}_{2}= K_{2} \frac{\pi d^{2}}{2} \exp \left[-\alpha_{2}\left(r^{2}+d^{2} / 8\right)\right] \cdot\left|W_{00}\left(r, f_{2}\right)\right| \cdot \\
& \sin \left[\beta_{2}\left(r^{2}+d^{2} / 8\right)+\varphi_{2}+\arg \left(W_{00}\left(r, f_{2}\right)\right)\right],
\end{aligned}
$$

and

$$
\begin{aligned}
\mathcal{C}_{3}=K_{3} \frac{\pi d^{2}}{2} \exp \left[-\alpha_{3}\left(r^{2}+d^{2} / 8\right)\right] \cdot\left|W_{00}\left(r, f_{3}\right)\right| \cdot \\
\cos \left[\beta_{3}\left(r^{2}+d^{2} / 8\right)-\varphi_{3}+\arg \left(W_{00}\left(r, f_{3}\right)\right)\right] .
\end{aligned}
$$

The parameters $f_{l}$ for $l=1,2,3$ are defined by:

$$
f_{l}=\frac{d^{2}}{4}\left(\beta_{l}+i \alpha_{l}\right)
$$

The function $W_{00}\left(r, f_{l}\right)$ can be expressed in Bessel-Bessel series such that [18]:

$$
W_{00}\left(r, f_{l}\right)=\sum_{k=0}^{\infty} A_{k}\left(f_{l}\right) \frac{J_{2 k+1}\left(4 f_{l} \frac{r}{d}\right)}{\left(4 f_{l} \frac{r}{d}\right)}
$$

with

$$
A_{k}\left(f_{l}\right)=(2 k+1)(-i)^{k} j_{k}\left(f_{l} / 2\right) .
$$

The function $j_{k}$ in Eq. (37) is the spherical Bessel function of the first kind and given by [19]:

$$
j_{k}(z)=\sqrt{\frac{\pi}{2 z}} J_{k+\frac{1}{2}}(z) .
$$

\subsection{Main results in general case $\left(z_{r} \neq z_{e}\right.$ and $\sigma$ finite) for the phase disk}

In this case, the result of the correlation can be expressed versus the correlation between the Gaussian chirplet and the intensity distribution for an opaque object, but here with an additional term, as one can see it in the previous section. Thus, one has:

$$
C_{a}\left(\mathcal{I}_{p}, \psi_{a}\right)=C_{a}\left(\mathcal{I}_{0}, \psi_{a}\right)-\sum_{l=1}^{3} \mathcal{D}_{l}
$$

with

$$
\begin{aligned}
\mathcal{D}_{1}= & K_{1} \frac{\pi d^{2}}{2} \exp \left[-\alpha_{1}\left(r^{2}+d^{2} / 8\right)\right] \cdot\left|W_{00}\left(r, f_{1}\right)\right| \cdot \\
& \sin \left[\beta_{1}\left(r^{2}+d^{2} / 8\right)-\left(\varphi_{1}-\varphi\right)+\arg \left(W_{00}\left(r, f_{1}\right)\right)\right] .
\end{aligned}
$$

Here again, the functions $\mathcal{D}_{2}$ and $\mathcal{D}_{3}$ are deduced from the structure of $\mathcal{D}_{1}$, then:

$$
\begin{aligned}
\mathcal{D}_{2}= & K_{2} \frac{\pi d^{2}}{2} \exp \left[-\alpha_{2}\left(r^{2}+d^{2} / 8\right)\right] \cdot\left|W_{00}\left(r, f_{2}\right)\right| \cdot \\
& \sin \left[\beta_{2}\left(r^{2}+d^{2} / 8\right)+\left(\varphi_{2}-\varphi\right)+\arg \left(W_{00}\left(r, f_{2}\right)\right)\right],
\end{aligned}
$$

and

$$
\begin{aligned}
\mathcal{D}_{3}=- & K_{3} \frac{\pi d^{2}}{2} \exp \left[-\alpha_{3}\left(r^{2}+d^{2} / 8\right)\right] \cdot\left|W_{00}\left(r, f_{3}\right)\right| \cdot \\
& \sin \left[\beta_{3}\left(r^{2}+d^{2} / 8\right)-\left(\varphi_{3}-\varphi\right)+\arg \left(W_{00}\left(r, f_{3}\right)\right)\right] .
\end{aligned}
$$

The theoretical estimation of the correlation product $C_{a}$ is then possible. A comparison between the numerical correlation by using the algorithm of FFT and theoretical correlation is given in Figure 4. The integral over spatial extension $x$ of the absolute difference of their intensities $\left|C_{a}\right|^{2}$ and $\left|\mathcal{F}^{-1}\left[\mathcal{F} I \times \mathcal{F} \psi_{a}\right]\right|^{2}$ gives us an error of $4.013 \times 10^{-5}$ for the opaque disk and $3.324 \times 10^{-5}$ for the phase disk. Then, we have shown Eqs. (31), (39) and (18) produce the same results. Numerical simulation is in good agreement with analytical results. This permits us to conclude that the theoretical developments of $C_{a}$ are right.

\subsection{Asymptotic behavior of the function $W_{00}$}

The important fact is that simulations of Eqs. (31) and (39) as shown in Figure 4 with $\mathcal{C}_{1}, \mathcal{C}_{2}$ and $\mathcal{C}_{3}$ are not always possible due to divergent behavior of the function $W_{00}$. Black corners on the reconstruction pattern shown in Figure 5(a) illustrate this point. 

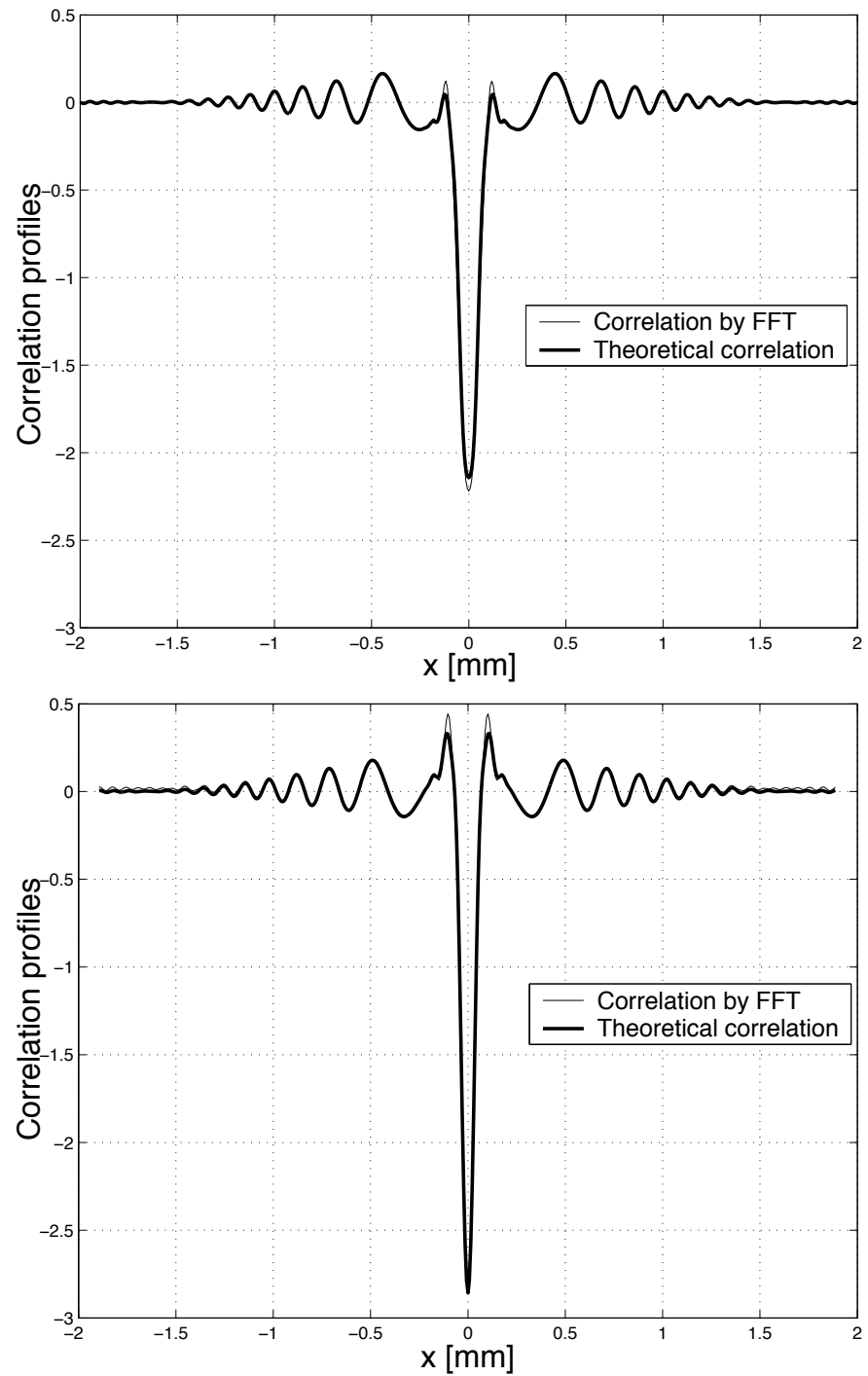

FIG. 4 Reconstruction in the best plane for an opaque and phase disk with $d=100 \mu \mathrm{m}$, $\lambda=635 \mathrm{~nm}, z_{e}=100 \mathrm{~mm}, z_{r}=110 \mathrm{~mm}, \sigma=6$.

The black corners are due to the overflows during the calculation. In the series for $W_{00}$, the term with $k=0$ is enough to explain the divergence of $W_{00}$ as $\left|4 f_{l} r / d\right|$ gets large. This is so, since the factor $j_{k}\left(f_{l} / 2\right)$, from 10.1 .2 on p. 437 of [19], decays very rapidly in $k$ and are all quite small except for $k=0$. Moreover, in the relevant range of $k$, all the factors $1 /\left(4 f_{l} r / d\right) \cdot J_{2 k+1}\left(4 f_{l} r / d\right)$ behave qualitatively the same. Thus the behavior of $\left|W_{00}\left(x, f_{l}\right)\right|$ for large $4 f_{l} r / d$ is very well given by:

$$
W_{00} \approx j_{0}\left(f_{l} / 2\right) \frac{J_{1}\left(\frac{4 f_{l}}{d} r\right)}{\frac{4 f_{l}}{d} r} \approx \frac{J_{1}\left(\frac{4 f_{l}}{d} r\right)}{\frac{4 f_{l}}{d} r} .
$$

Next by using Hankel's asymptotic expansions (9.2.5, p. 364 of [19]), for the Bessel functions:

$$
\begin{aligned}
J_{v}(\zeta) \approx \sqrt{\frac{2}{\zeta \pi}}[(1 & \left.+O\left(\zeta^{-2}\right)\right) \cos (\chi) \\
& \left.-\frac{4 v^{2}-1}{8 \zeta}\left(1+O\left(\zeta^{-2}\right)\right) \sin (\chi)\right]
\end{aligned}
$$

with $\chi=\zeta-(v / 2+1 / 4) \pi$ and $\zeta=4 f_{l} r / d$. In this example, the values of $|\zeta|^{2}$ grows from 7936 to 31745 . Thus we may ignore the $O\left(\zeta^{-2}\right)$ in Eq. (44). By noting that the $\Im\{\chi\}=\Im\{\zeta\}$ and that $\Im\{\zeta\}$ grows from 89.06 to $178, \exp (i \chi)$ can neglected
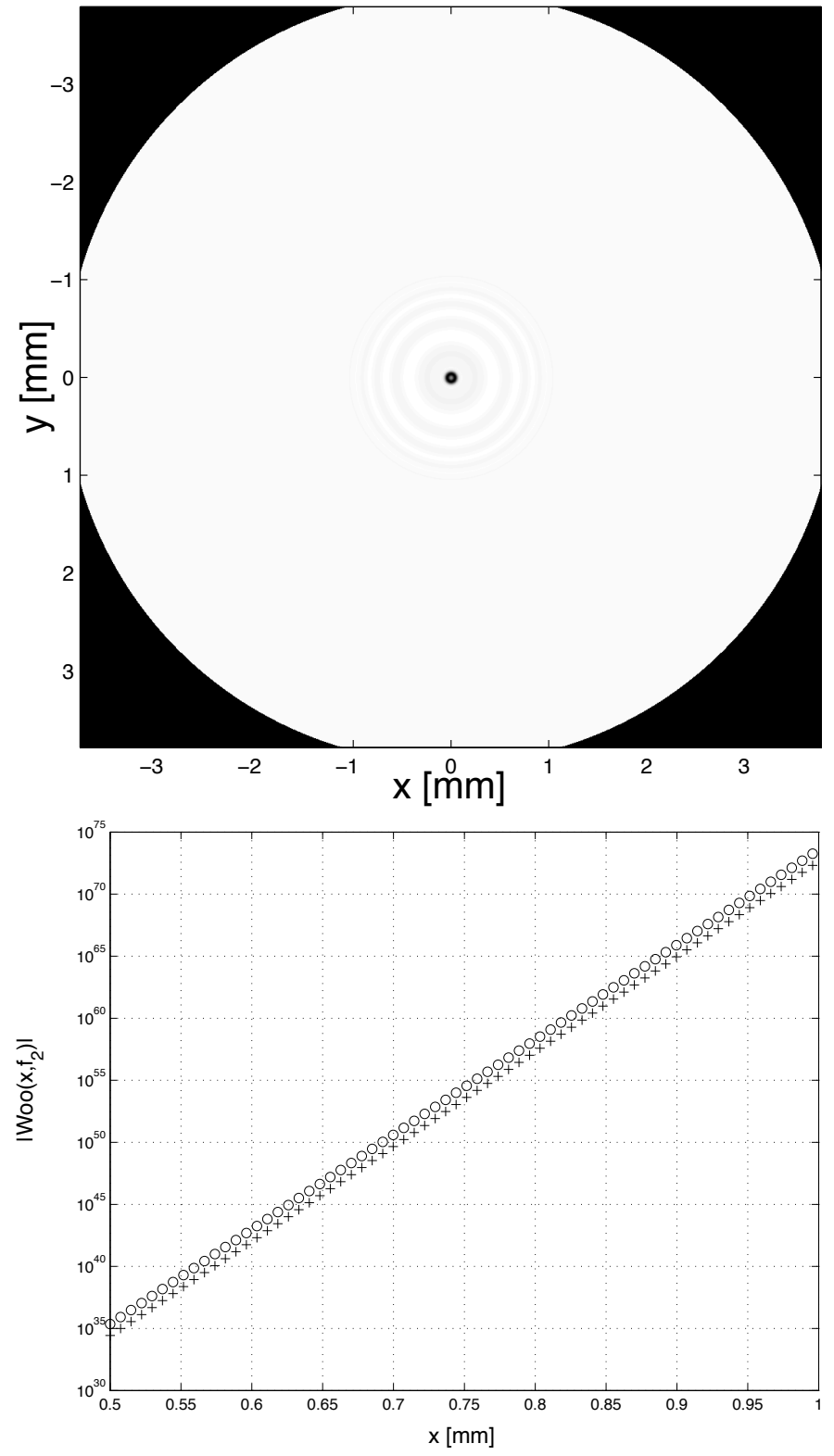

FIG. $5 d=100 \mu \mathrm{m}, \lambda=635 \mathrm{~nm}, z_{e}=100 \mathrm{~mm}, z_{r}=100.01 \mathrm{~mm}, \sigma=6, f_{2} \simeq-0.108+$ 4.468i. (a) reconstruction pattern of an opaque disk, (b) semilog representation of $\left|W_{00}\left(x, f_{2}\right)\right|$.

in comparison to $\exp (-i \chi)$ when $\Im\{\zeta\}$ grows large. Then it follows that:

$$
J_{v}(\zeta) \approx \frac{1}{2} \sqrt{\frac{2}{\zeta \pi}}\left[1+\frac{4 v^{2}-1}{8 i \zeta}\right] \exp (-i \chi) .
$$

One can conclude that the behavior of $\left|W_{00}\right|$ for large $\left|4 f_{l} r / d\right|$ is approximatively equal to:

$$
\left|W_{00}\right| \approx\left|\frac{J_{1}(\zeta)}{\zeta}\right|=\frac{\exp \left[\alpha_{l} d r\right]}{\left|4 f_{l} \frac{r}{d}\right|^{3 / 2} \sqrt{2 \pi}} .
$$

Figure 5(b) allows us to compare the approximation Eq. (46) with Eq. (36) in cross markers. This representation is given in semi-logarithmic scale. As $W_{00}$ is versus exponential function in Eq. (46) then a linear function is obtained. Note however that this divergence does not act upon the correlation result because Gaussian function in Eqs. (32)-(34) associated with Eq. (46) gives us a convergent function to $r$-infinity. The calculation of $C_{a}$ with Eq. (18) does not produce an overflow. Nevertheless, the digital simulations of $C_{l}$ with $l=1,2,3$ must 




FIG. 6 Influence of $\sigma$ on $\left|W_{00}\left(x, f_{2}\right)\right|$ for $d=100 \mu \mathrm{m}, z_{e}=100 \mathrm{~mm}, z_{r}=110 \mathrm{~mm}$.

be realized without any difficulty, provided that all functions $C_{l}$ are asymptotical convergent. Therefore, this behavior depends on the choice of the chirplet function $\psi_{a}$, i.e. $\alpha_{l}$. The correlation product between the intensity distribution $I(\mathbf{r})$ and the Gaussian chirplet function $\psi_{a}(\mathbf{r})$ implies that one of the functions that describes the correlation function is divergent. This is due to the presence of exponential functions versus $\alpha_{l}$, for $l=1,2,3$ and defined by:

$$
\alpha_{l}=\frac{1}{\sigma^{2} M_{l}^{2} a^{2}} .
$$

To obtain convergent functions that describe the solution of the correlation product, it is necessary that the parameter $\alpha_{l}$ is equal to zero. In this case, Eq. (46) proves that the function $\left|W_{00}\left(x, f_{l}\right)\right|$ tends to zero when $r$ tends to infinity. The condition $\alpha_{l}=0$ is realized when $\sigma$ tends to infinity. The influence of the $\sigma$ on the divergence of $\left|W_{00}\right|$ is illustrated in Figure 6. As one can see, a greater $\sigma$ corresponds to a larger $\left|W_{00}\left(x, f_{2}\right)\right|$.

Consequently, the chirplet function should have the following form

$$
\psi_{a}(\mathbf{r})=\frac{1}{a^{2}} \sin \left(\frac{r^{2}}{a^{2}}\right),
$$

to assure the convergence of all functions that describe the correlation result $C_{a}$. Note that this function is closed to the fractional Fourier transformation when $\sigma \longrightarrow \infty$ and $a=s^{2} \tan \alpha$ [13] or closed to the imaginary part of Fresnel's integral kernel. Under this condition, all functions are convergent and stable on $\mathbb{R}^{2}$. Figures $7(\mathrm{a})$ and (b) represents the profile of the correlation product for an opaque and phase disk. The profile shown with a dashed line is obtained when $\sigma$ is finite, and the solid profile when $\sigma$ tends to infinity. As one can see, the proposition $\sigma$ infinite allows a profile close to the object to be obtained. The edges are more abrupt. The transition band are smallest without modifying the general dynamic of the signal.

Nevertheless, in the case even where the mathematical definition of the chirplet Gaussian function Eq. (17) must be preserved, it is possible to consider the following approximate relation:

$$
a \sigma \gg \frac{\pi^{2}}{M_{l}}[d \cdot \max (r)]^{1 / 2}
$$
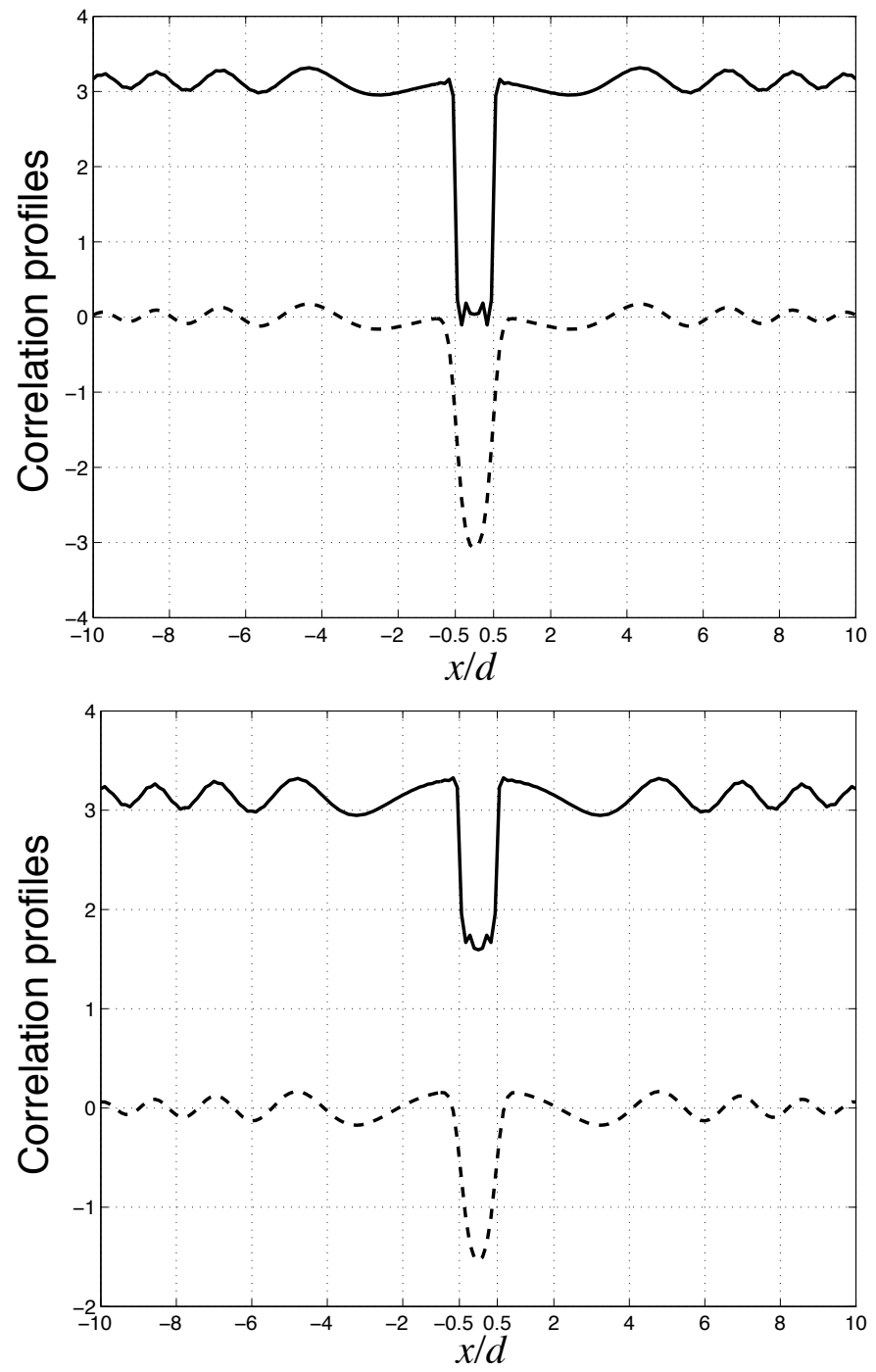

FIC. 7 Profile of the correlation products with the modified chirplet function (solid line, $\sigma \longrightarrow \infty$ ) and with the original chirplet function (dashed line, $\sigma=6$ ). $d=100 \mu \mathrm{m}$, $\lambda=635 \mathrm{~nm}, z_{e}=z_{r}=100 \mathrm{~mm}$, (a) opaque disk, (b) phase disk.

to assure that the value of $\alpha_{l} \ll 1$.

\subsection{Particular case of the reconstruction of image of the object}

From the results in Section 2.2 and by considering the Dirac delta function $\delta(r)$ as the limit of parametric continuous function:

$$
\delta(r)=\lim _{c \rightarrow 0} \frac{1}{|c|} \exp \left[-\pi \frac{r^{2}}{|c|}\right]
$$

the particular case where $\sigma$ tends to infinity gives us the following functions:

$$
\lim _{\substack{z e \\ z r \rightarrow 1 \\ \sigma \rightarrow \infty}} \mathcal{K}_{o}=-\delta(\mathbf{r})-\frac{1}{2 a^{2}} \sin \left[\frac{r^{2}}{2 a^{2}}\right]
$$

and

$$
\lim _{\substack{z_{e} \rightarrow 1 \\ z_{r} \rightarrow \infty}} \mathcal{L}_{p}=-\delta(\mathbf{r}) \cos (\varphi)-\frac{1}{2 a^{2}} \sin \left[\frac{r^{2}}{2 a^{2}}+\varphi\right]
$$


Finally, the relation $C_{a}\left(\mathcal{I}_{0}, \psi_{a}\right)$ becomes:

$$
\lim _{\substack{z e \\ \frac{z}{z} \rightarrow 1 \\ \sigma \rightarrow \infty}} C_{a}\left(\mathcal{I}_{o}, \psi_{a}\right)=-\Pi(\mathbf{r})-\Pi(\mathbf{r}) * \frac{1}{2 a^{2}} \sin \left[\frac{r^{2}}{2 a^{2}}\right],
$$

and $C_{a}\left(\mathcal{I}_{p}, \psi_{a}\right)$

$$
\begin{aligned}
\lim _{\substack{z_{e} \rightarrow 1 \\
z_{r} \rightarrow \infty \\
\sigma \rightarrow \infty}} C_{a}\left(\mathcal{I}_{p}, \psi_{a}\right)=-(1-\cos (\varphi)) \Pi(\mathbf{r}) \\
\quad-\Pi(\mathbf{r}) * \frac{1}{2 a^{2}}\left[\sin \left(\frac{r^{2}}{2 a^{2}}\right)-\sin \left(\frac{r^{2}}{2 a^{2}}+\varphi\right)\right] .
\end{aligned}
$$

Eqs. (53) and (54) exhibit the transmittance $\Pi(\mathbf{r})$ which makes up the object. The first term of Eqs. (53) and (54) is the unit circular function with an aperture equal to the diameter of the object. The minus sign before $\Pi$ allows the same transmittance that the object to be obtained in a gray level representation. The second term generally corresponds to the virtual image in classical holography. Choosing $\sigma$ infinite allows the numerical recording-reconstruction process $C_{a}$ to refocus on the object. The best focus plane is then obtained with $\sigma \rightarrow \infty$ and $z_{r} \rightarrow z_{\mathcal{e}}$.

\section{CONCLUSION}

In this paper, an analytical solution of digital in-line holography from correlation with a chirplet function is proposed. The analytical correlation demonstrates that the recordingreconstruction process does not allow an optical refocusing on the image of the object but allows us to obtain a maximum likelihood between the chirplet function and the intensity distribution of the diffraction pattern. A proposition of the chirplet function is given to resolve the optical refocusing question. With this proposition, the transmittance of the objet appears clearly in the mathematical relations.

\section{APPENDIX}

\section{A DEFINITION OF THE FUNCTIONS $T_{1}$ TO $T_{5}$}

Let us recall an important integral which is frequently used in this paper [20]:

$$
\int \exp \left[-p^{2} x^{2} \pm i 2 \pi u x\right] d x=\frac{\sqrt{\pi}}{p} \exp \left(-\frac{\pi^{2}}{p^{2}} u^{2}\right) .
$$

Note that the choice of the definition of the Fourier transformation is the following:

$$
\mathcal{F}[f](u, v)=\iint f(x, y) \exp (-i 2 \pi(u x+v y)) d x d y,
$$

where $u, v$ define the spatial frequencies.

\section{A.1 Definition of the function $T_{1}$}

The function $T_{1}$ is the result of a convolution product and it is well known that the convolution of two functions means multiplication of their transforms. Thus:

$$
\mathcal{F}\left[T_{1}\right](u, v)=\mathcal{F}\left[h_{z_{e}}\right](u, v) \times \mathcal{F}\left[g h_{z_{r}}\right](u, v) .
$$

The Fourier transform of $h_{z_{e}}$ by using Eq. (A.1) gives us:

$$
\mathcal{F}\left[h_{z_{e}}\right](u, v)=i \lambda z_{e} \exp \left(-i \pi \lambda z_{e}\left(u^{2}+v^{2}\right)\right),
$$

and

$$
\mathcal{F}\left[g h_{z_{r}}\right](u, v)=\frac{\pi}{\gamma_{1}^{2}} \exp \left(-\frac{\pi^{2}}{\gamma_{1}^{2}}\left(u^{2}+v^{2}\right)\right),
$$

with $\gamma_{1}^{2}=\left(1 / \sigma^{2}-i\right) / a^{2}$. Finally, the Fourier transform of $T_{1}$ is:

$$
\mathcal{F}\left[T_{1}\right](u, v)=i \lambda z_{e} \frac{\pi}{\gamma_{1}^{2}} \exp \left(-\eta_{1}^{2}\left(u^{2}+v^{2}\right)\right)
$$

with $\eta_{1}^{2}=\pi^{2} / \gamma_{1}^{2}+i \pi \lambda z_{e}$. The inverse Fourier transformation of Eq. (A.6) gives us the definition of $T_{1}$, i.e.:

$$
\begin{aligned}
T_{1}(x, y)=i \lambda z_{e} \frac{1}{M_{1}} & \exp \left[-\frac{1}{\sigma^{2} M_{1}^{2}} \frac{r^{2}}{a^{2}}\right] \\
& \times \exp \left[i \frac{1}{M_{1}^{2}}\left(1+\frac{z_{e}}{z_{r}}\left(1+1 / \sigma^{4}\right)\right) \frac{r^{2}}{a^{2}}-i \varphi_{1}\right],
\end{aligned}
$$

with

$$
M_{1}=\left|\left(1+\frac{z_{e}}{z_{r}}\right)+i \frac{1}{\sigma^{2}} \frac{z_{e}}{z_{r}}\right|,
$$

and

$$
\varphi_{1}=\arctan \left(\frac{1}{\sigma^{2}} \frac{z_{e} / z_{r}}{1+z_{e} / z_{r}}\right)
$$

\section{A.2 Definition of the function $T_{2}$}

In the same way as the elaboration of $T_{1}$, we obtain:

$$
\begin{aligned}
T_{2}(x, y)=i \lambda z_{e} \frac{1}{M_{2}} \exp \left[-\frac{1}{\sigma^{2} M_{2}^{2}} \frac{r^{2}}{a^{2}}\right] \\
\exp \left[-i \frac{1}{M_{2}^{2}}\left(1-\frac{z_{e}}{z_{r}}\left(1+1 / \sigma^{4}\right)\right) \frac{r^{2}}{a^{2}}-i \varphi_{2}\right],
\end{aligned}
$$

with

$$
M_{2}=\left|\left(1-\frac{z_{e}}{z_{r}}\right)+i \frac{1}{\sigma^{2}} \frac{z_{e}}{z_{r}}\right|,
$$

and

$$
\varphi_{2}=\arctan \left(\frac{1}{\sigma^{2}} \frac{z_{e} / z_{r}}{1-z_{e} / z_{r}}\right)
$$

If $\left(1-z_{e} / z_{r}\right)<0$ then, to assure the continuity of the function, the phase $\pi$ is added to $\varphi_{2}$ such as:

$$
\varphi_{2}=\arctan \left(\frac{1}{\sigma^{2}} \frac{z_{e} / z_{r}}{1-z_{e} / z_{r}}\right)+\pi
$$

The additional phase $\pi$ corresponds to the Gouy phase shift that arises when Gaussians propagate from $-\infty$ to $+\infty$ through the focus point. 


\section{A.3 Definition of $T_{3}$}

The Fourier transform of $T_{3}$ is:

$$
\mathcal{F}\left[T_{3}\right](u, v)=\mathcal{F}\left[\bar{h}_{z_{e}}\right](u, v) \times \mathcal{F}\left[g h_{z_{r}}\right](u, v) .
$$

By means of the property:

$\mathcal{F}\left[\bar{h}_{z_{e}}\right](u, v)=\overline{\mathcal{F}\left[h_{z_{e}}\right]}(u, v)=-i \lambda z_{e} \exp \left(i \pi \lambda z_{e}\left(u^{2}+v^{2}\right)\right)$,

the definition of $T_{3}$ is obtained as:

$$
\begin{aligned}
T_{3}(x, y)=- & i \lambda z_{e} \frac{1}{M_{2}} \exp \left[-\frac{1}{\sigma^{2} M_{2}^{2}} \frac{r^{2}}{a^{2}}\right] \\
& \times \exp \left[i \frac{1}{M_{2}^{2}}\left(1-\frac{z_{e}}{z_{r}}\left(1+1 / \sigma^{4}\right)\right) \frac{r^{2}}{a^{2}}+i \varphi_{2}\right] .
\end{aligned}
$$

\section{A.4 Definition of $T_{4}$}

The Fourier transform of $T_{4}$ is given by:

$$
\mathcal{F}\left[T_{4}\right](u, v)=F\left[\bar{h}_{z_{e}}\right](u, v) \times \mathcal{F}\left[g \bar{h}_{z_{r}}\right](u, v) .
$$

The second expression of Eq. (A.17), next few simple calculus, holds:

$$
\mathcal{F}\left[g \bar{h}_{z_{r}}\right](u, v)=\frac{\pi}{\gamma_{4}^{2}} \exp \left(-\frac{\pi^{2}}{\gamma_{4}^{2}}\left(u^{2}+v^{2}\right)\right),
$$

with $\gamma_{4}^{2}=\left(1 / \sigma^{2}+i\right) a^{2}$. By considering all previous results of $T_{l}, l=1,2,3$, finally $T_{4}$ takes the following definition:

$$
\begin{aligned}
& T_{4}(x, y)=- i \lambda z_{e} \frac{1}{M_{1}} \exp \left[-\frac{1}{\sigma^{2} M_{1}^{2}} \frac{r^{2}}{a^{2}}\right] \\
& \times \exp \left[-i \frac{1}{M_{1}^{2}}\left(1+\frac{z_{e}}{z_{r}}\left(1+1 / \sigma^{4}\right)\right) \frac{r^{2}}{a^{2}}+i \varphi_{1}\right] .
\end{aligned}
$$

\section{A. 5 Definition of $T_{5}$}

Again, by using the Fourier operator and its property on the convolution product, we have:

$$
\begin{aligned}
&\left(h_{z_{e}}-\bar{h}_{z_{e}}\right) * g(\mathbf{r})= i 2 \lambda z_{e} \frac{1}{M_{3}} \exp \left[-\frac{1}{\sigma^{2} M_{3}^{2}} \frac{r^{2}}{a^{2}}\right] \\
& \cos \left[\frac{1}{\sigma^{4} M_{3}^{2}} \frac{z_{e}}{z_{r}} \frac{r^{2}}{a^{2}}-\arctan \left(\frac{1}{\sigma^{2}} \frac{z_{e}}{z_{r}}\right)\right] .
\end{aligned}
$$

\section{B DEFINITION OF $C_{1}$}

Owing to the radial symmetry of the functions, it is convenient to restate $C_{a}$ in cylindrical coordinates as follows: $x=r \cos \theta$, $y=r \sin \theta$ and $x^{\prime}=\rho \cos \phi, y^{\prime}=\rho \sin \phi$, so that:

$$
\begin{aligned}
C_{a}=\int_{0}^{2 \pi} \int_{0}^{+\infty} & \Pi(\rho \cos \phi, \rho \sin \phi) \\
& \times \mathcal{K}_{o}(r \cos \theta-\rho \cos \phi, r \sin \theta-\rho \sin \phi) \rho d \rho d \phi .
\end{aligned}
$$

The function $\Pi$ is the circular function of aperture $d$, then $\Pi(\rho \cos \phi, \rho \sin \phi)=\Pi(\rho)$. All the other functions, i.e. $\mathcal{K}_{o}$ and $F_{l}$ are versus $r^{2}$ then the coordinates in Eq. (B.1) become:

$$
\left(x-x^{\prime}\right)^{2}+\left(y-y^{\prime}\right)^{2}=r^{2}+\rho^{2}-2 r \rho \cos (\phi-\theta) .
$$

By taking into account the expression of the functions $F_{k}$ and by expanding their expressions to complex exponential functions, yields,

$$
\begin{aligned}
& F_{1}(r, \theta, \rho, \phi)=\frac{K_{1}}{2 i}\left[s_{1+}+s_{1-}\right], \\
& F_{2}(r, \theta, \rho, \phi)=\frac{K_{2}}{2 i}\left[s_{2+}+s_{2-}\right],
\end{aligned}
$$

and

$$
F_{3}(r, \theta, \rho, \phi)=\frac{K_{3}}{2}\left[s_{3+}+s_{3-}\right]
$$

with

$s_{1 \pm}= \pm \exp \left[-\left(\alpha_{1} \mp i \beta_{1}\right)\left(r^{2}+\rho^{2}-2 r \rho \cos (\phi-\theta)\right)\right] \exp \left[\mp i \varphi_{1}\right]$,

$s_{2 \pm}= \pm \exp \left[-\left(\alpha_{2} \mp i \beta_{2}\right)\left(r^{2}+\rho^{2}-2 r \rho \cos (\phi-\theta)\right)\right] \exp \left[ \pm i \varphi_{2}\right]$,

and

$$
s_{3 \pm}=\exp \left[-\left(\alpha_{3} \mp i \beta_{3}\right)\left(r^{2}+\rho^{2}-2 r \rho \cos (\phi-\theta)\right)\right] \exp \left[\mp i \varphi_{3}\right] .
$$

It is sufficient to expand the correlation product, denoted $c_{1 \pm}$, of $s_{1 \pm}$ by $\Pi$ to deduce the other relations, then:

$$
\begin{aligned}
c_{1 \pm}= & \int_{0}^{2 \pi} \int_{0}^{+\infty} \Pi(\rho) . \\
& \times s_{1 \pm}(r \cos \theta-\rho \cos \phi, r \sin \theta-\rho \sin \phi) \rho d \rho d \phi
\end{aligned}
$$

with

$$
C_{1}=c_{1+}+c_{1-}
$$

We have:

$$
\begin{aligned}
c_{1 \pm} & =\int_{0}^{2 \pi} \int_{0}^{d / 2} \pm \frac{K_{1}}{2 i} \\
& \times \exp \left[-\left(\alpha_{1} \mp i \beta_{1}\right)\left(r^{2}+\rho^{2}-2 r \rho \cos (\phi-\theta)\right) \mp i \varphi_{1}\right] \rho d \rho d \phi .
\end{aligned}
$$

By using the relation [19]:

$$
\frac{1}{2 \pi} \int_{0}^{2 \pi} \exp (i n \theta) \exp [i x \cos \theta] d \theta=i^{n} J_{n}(x),
$$

we obtain:

$$
\begin{aligned}
c_{1 \pm}= \pm & 2 \pi \frac{K_{1}}{2 i} \exp \left[-\left(\alpha_{1} \mp i \beta_{1}\right) r^{2} \mp i \varphi_{1}\right] \\
& \times \int_{0}^{d / 2} \exp \left[-\left(\alpha_{1} \mp i \beta_{1}\right) \rho^{2}\right] J_{0}\left[2\left(\mp \beta_{1}-i \alpha_{1}\right) r \rho\right] \rho d \rho .
\end{aligned}
$$

By writing $f_{1 \pm}=d^{2}\left( \pm \beta_{1}+i \alpha_{1}\right) / 4$ and by normalizing $\rho$ by $d / 2$, the structure of $c_{1}$ becomes:

$$
\begin{aligned}
c_{1 \pm}= \pm & 2 \pi \frac{K_{1}}{2 i} \exp \left[i 4 f_{1 \pm} \frac{r^{2}}{d^{2}} \mp i \varphi_{1}\right] \frac{d^{2}}{4} \\
& \times \int_{0}^{1} \rho \exp \left[i f_{1 \pm} \rho^{2}\right] J_{0}\left[\left(4 f_{1 \pm} \frac{r}{d}\right) \rho\right] d \rho .
\end{aligned}
$$


In Eq. (B.14), we considered that Bessel function of zero order is an even function: $J_{0}(-z)=J_{0}(z)$. By knowing that:

$$
V_{n m}(r, f)=\int_{0}^{1} \exp \left(i f \rho^{2}\right) R_{n}^{m}(\rho) J_{m}(2 \pi r \rho) \rho d \rho,
$$

where $R_{n}^{m}$ is the circle polynomials of Zernike [2] with $R_{0}^{0}=1$, Eq. (B.15) can be expressed in the Bessel-Bessel series expression such as [18]:

$$
\begin{aligned}
V_{n m}(r, f)= & \exp \left(\frac{1}{2} \text { if }\right) \sum_{k=0}^{\infty}(2 k+1)(i)^{k} j_{k}(f / 2) \\
& \cdot \sum_{l=\max (0, k-q, p-k)}^{k+p}(-1)^{l} \omega_{k l} \frac{J_{m+2 l+1}(2 \pi r)}{(2 \pi r)} .
\end{aligned}
$$

By comparing Eqs. (B.15) and (B.16), then $n=m=0$ so that $p=q=0$ and $l=k$. The coefficient $\omega_{k l}=1$, the equation of $c_{1 \pm}$ can be then expressed as:

$$
c_{1 \pm}= \pm \frac{K_{1}}{2 i} \exp \left[i 4 f_{1 \pm} \frac{r^{2}}{d^{2}} \mp i \varphi_{1}\right] \frac{\pi d^{2}}{2} V_{00}\left(r, f_{1 \pm}\right) .
$$

with

$$
V_{00}(r, f)=\exp \left(\frac{1}{2} i f\right) \sum_{k=0}^{\infty} A_{k}(f) \frac{J_{2 k+1}\left(4 f \frac{r}{d}\right)}{\left(4 f \frac{r}{d}\right)},
$$

and

$$
A_{k}(f)=(2 k+1)(-i)^{k} j_{k}(f / 2) .
$$

The function $j_{k}$ is the spherical Bessel function of the first kind and is given by [19]:

$$
j_{k}(z)=\sqrt{\frac{\pi}{2 z}} J_{k+\frac{1}{2}}(z) .
$$

By means of the relations 10.1 .34 on p. 439 and 9.1 .40 on p. 361 of [19]:

$$
j_{n}(z \exp [i m \pi])=\exp [m n i \pi] j_{n}(z), \quad J_{n}(\bar{z})=\overline{J_{n}(z)},
$$

the correlation function $C_{1}$, in Eq. (B.10), can be obtained.

\section{Acknowledgments}

The authors thank their colleagues Dr. P. Janssen from Philips Reseach Leuven for his help and thank reviewers for their comments.

\section{References}

[1] S. Mann, and S. Haykin, "The chirplet transform: physical considerations" IEEE T. Signal Proces. 43, 2745-2761 (1995).

[2] M. Born, and E. Wolf, Principles of Optics (7th edition, Cambridge University Press, Cambridge, 1999).

[3] J. W. Goodman, and R. W. Lawrence, "Digital image formation from electronically detected holograms" Appl. Phys. Lett. 11, 77 (1967).

[4] L. Onural, and M. Kocatepe, "Family of scaling chirp functions, diffraction, and holography" IEEE T. Signal Proces. 43, 1568-1578 (1995).

[5] L. Cheng, "Simultaneous measurement of displacement and its spatial derivatives with a digital holographic method" Opt. Eng. 42, 3443-3446 (2003).

[6] D. Cabor, "A new microscopic principle" Nature 61, 777-778 (1948).

[7] J. W. Goodman, Introduction to Fourier Optics (3rd edition, Roberts and Company Publishers, Colorado, 2005).

[8] U. Schnars and W. Jüptner, "Direct recording of holograms by a CCD target and numerical reconstruction" Appl. 0pt. 33, 179-181 (1994).

[9] F. Slimani, G. Grehan, G. Gouesbet, and D. Allano, “Near-field Lorenz-Mie theory and its application to microholography" Appl. Opt. 23, 4140-4148 (1984).

[10] F. Nicolas, S. Coëtmellec, M. Brunel, and D. Lebrun, "Suppression of the Moiré effect in sub-picosecond digital in-line holography" Opt. Express 15, 887-895 (2007).

[11] M. Brunel, S. Coëtmellec, D. Lebrun, and K. A. Ameur, "Digital phase contrast with the fractional Fourier transform" Appl. 0pt. 48, 579-583 (2009).

[12] C. Buraga-Lefebvre, S. Coëtmellec, D. Lebrun, and C. Özkul, "Application of wavelet transform to hologram analysis: threedimensional location of particles" Opt. Lasers Eng. 33, 409-421 (2000).

[13] S. Coëtmellec, D. Lebrun, and C. Özkul, "Application of the twodimensional fractional-order Fourier transformation to particle field digital holography" J. Opt. Soc. Am. A. 19, 1537-1546 (2002).

[14] M. Malek, S. Coëtmellec, D. Lebrun, and D. Allano, "Formulation of in-line holography process by a linear shift invariant system: Application to the measurement of fiber diameter" Opt. Commun. 223, 263-271 (2003).

[15] L. Onural, and P. D. Scott, "Digital decoding of in-line holograms" Opt. Eng. 26, 1124-1132 (1987).

[16] F. Dubois, C. Schockaert, N. Callens, and C. Yourassowsky, "Focus plane detection criteria in digital holography microscopy by amplitude analysis" Opt. Express 14, 5895-5908 (2006).

[17] W. Li, N. C. Loomis, Q. Hu, and C. S. Davis, "Focus detection from digital in-line holograms based on spectral 11 norms" J. Opt. Soc. Am. A. 24, 3054-3062 (2007).

[18] A. J. E. M. Janssen, J. J. M. Braat, and P. Dirksen, "On the computation of the Nijboer-Zernike aberration integrals at arbitrary defocus" J. Mod. Opt. 51, 687-703 (2004).

[19] M. Abramowitz, and I. A. Stegun, Handbook of Mathematical Functions (Dover Publications Inc., New York, 1970).

[20] H. M. Ozaktas, Z. Zalevsky, and M. A. Kutay, The Fractional Fourier Transform with Applications in Optics and Signal Processing (1st edition, Wiley, England, 2001). 